\title{
Numerical modeling of laser assisted tape winding process
}

Amin Zaami, Ismet Baran, and Remko Akkerman

Citation: AIP Conference Proceedings 1896, 180004 (2017); doi: 10.1063/1.5008209

View online: http://dx.doi.org/10.1063/1.5008209

View Table of Contents: http://aip.scitation.org/toc/apc/1896/1

Published by the American Institute of Physics 


\title{
Numerical Modeling of Laser Assisted Tape Winding Process
}

\author{
Amin Zaami ${ }^{1, a)}$, Ismet Baran, ${ }^{1 \text { b) }}$, Remko Akkerman ${ }^{1, c)}$, \\ ${ }^{1}$ Chair of Production Technology, Faculty of Engineering Technology, University of Twente, 7500AE, Enschede, \\ The Netherlands \\ a)a.zaami@utwente.nl

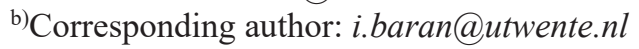 \\ c)r.akkerman@utwente.nl
}

\begin{abstract}
Laser assisted tape winding (LATW) has become more and more popular way of producing new thermoplastic products such as ultra-deep sea water riser, gas tanks, structural parts for aerospace applications. Predicting the temperature in LATW has been a source of great interest since the temperature at nip-point plays a key role for mechanical interface performance. Modeling the LATW process includes several challenges such as the interaction of optics and heat transfer. In the current study, numerical modeling of the optical behavior of laser radiation on circular surfaces is investigated based on a ray tracing and non-specular reflection model. The non-specular reflection is implemented considering the anisotropic reflective behavior of the fiber-reinforced thermoplastic tape using a bidirectional reflectance distribution function (BRDF). The proposed model in the present paper includes a three-dimensional circular geometry, in which the effects of reflection from different ranges of the circular surface as well as effect of process parameters on temperature distribution are studied. The heat transfer model is constructed using a fully implicit method. The effect of process parameters on the nip-point temperature is examined. Furthermore, several laser distributions including Gaussian and linear are examined which has not been considered in literature up to now.
\end{abstract}

Keywords. LATW, Reflection, Thermoplastic composite, Numerical modeling

\section{INTRODUCTION}

Advanced manufacturing processes of composite materials have been developed in recent years to achieve improved mechanical properties. Among these processes, the laser assisted tape winding (LATW) which is an automated process to produce tubular or tube-like parts has been a source of great interest to attain higher accuracy, repeatability, quality and reducing cost cycle time [1]. This process has been studied in different disciplines from experimental studies to simulations employing different simplifying assumptions [2-5]. During the process, the material which is usually unidirectional reinforced thermoplastic material, is heated by a laser and pressure is applied for the consolidation by a roller. The optimum process conditions which can be defined by optical and thermal models are needed to ensure good consolidation and adhesion between layers.

Several studies have been carried out to model the LATW or laser assisted tape placement (LATP) processes considering coupled optical and thermal models [2,6]. A numerical process model was developed in [2] to predict the temperature distribution in the tape and laminate for the LATP in which a ray-tracing method was used together with a one-dimensional (1D) thermal model. Stokes-Griffin [6] developed a more detailed model in 3D including the actual laser power distribution. The composite surface was modeled as a collection of micro-half-cylinders. Both aforementioned optical models dealt with flat substrates. However, when a cylindrical substrate is used with different geometrical parameters, a more complicated reflection model should be taken into account. It can play an important role on the energy absorption/reflection and subsequently temperature distribution. In this regard, there is a lack of parameter study regarding the effects of geometry on the specular and non-specular reflection behavior in literature. Besides, different laser distributions cause the reflection behavior of the composite to be more complicated, and somewhat makes it difficult to predict the nip-point temperature. Thus, quantitative analysis is necessary to understand the influence of the geometrical effects and laser patterns on the reflection and temperature.

In this study, first overall schematic view of the process with parameters are defined. Then, four different laser distributions are introduced with the same input laser power to heat the tape and laminate surfaces. The non-specular reflection model (BRDF) developed in [7] is used which is capable of simulating the anisotropic reflection behavior. The effect of laser distribution on the nip-point temperature and reflection behavior is quantified. 


\section{MATERIALS AND MODEL}

This section introduces different laser distributions and how optical-thermal model works. The user provides a set of input parameters that are sent to the optical model (detailed in [7]). The output of the optical model is a twodimensional laser irradiation field on each surface that describes the heat flux (laser power per unit area). This information is used by the thermal model to calculate the temperature. The geometrical parameters together with the schematic view are presented in Fig.1. It should be mentioned that the roller and mandrel are considered in y-z plane in the simulation as seen in Fig. 1.

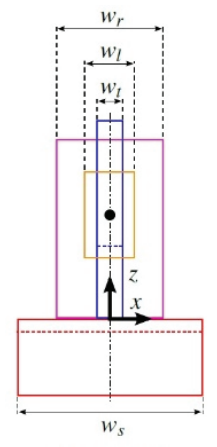

Front view

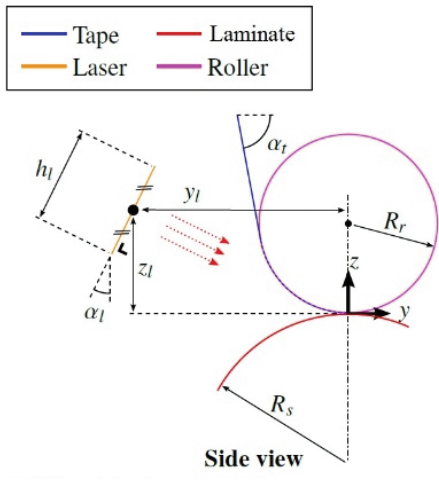

Side view

\begin{tabular}{|l|c|}
\hline Laser $(\mathrm{y}, \mathrm{z})(\mathrm{mm})$ & $-300,80$ \\
\hline Beam height $\boldsymbol{h}_{\boldsymbol{l}}(\mathrm{mm})$ & 30 \\
\hline Beam width $\boldsymbol{w}_{\boldsymbol{l}}(\mathrm{mm})$ & 12 \\
\hline Angle $\boldsymbol{\alpha}_{\boldsymbol{l}}(\mathrm{deg})$ & -14 \\
\hline Roller $(\mathrm{y}, \mathrm{z})(\mathrm{mm})$ & 0,34 \\
\hline Roller radius $\boldsymbol{R}_{\boldsymbol{r}}(\mathrm{mm})$ & 34 \\
\hline Roller width $\boldsymbol{w}_{\boldsymbol{r}}(\mathrm{mm})$ & 50 \\
\hline Tape width $\boldsymbol{w}_{\boldsymbol{t}}(\mathrm{mm})$ & 6 \\
\hline Tape angle $\boldsymbol{\alpha}_{\boldsymbol{t}}(\mathrm{deg})$ & -60 \\
\hline Laminate width $\boldsymbol{w}_{\boldsymbol{l}}(\mathrm{mm})$ & 20 \\
\hline Laminate radius $\boldsymbol{R}_{\boldsymbol{s}}(\mathrm{mm})$ & 34 \\
\hline
\end{tabular}

FIGURE 1. Geometrical parameters of the optical model.

The winding speed is set to $150 \mathrm{~mm} / \mathrm{s}$, the laminate thickness is considered as $2.5 \mathrm{~mm}$ and tape thickness is 0.25 $\mathrm{mm}$. For each geometrical shape, aspect ratio is defined as the ratio of laminate radius to the roller radius as $\mathrm{AR}=R_{S}$ $/ R_{r}$. In addition, different laser distributions are defined in a specific area between $\left[-\xi_{0}, \xi_{0}\right]$ and $\left[-\eta_{0}, \eta_{0}\right]$, in which $\xi$ is the width direction (x-direction) and $\eta$ is the direction of placement (y-direction). Two cases (Top-hat and Gaussian) are considered with a gradual decrease of intensity outside the boundaries and the other two cases (linear width skewness and linear longitudinal skewness) are assumed to have perfectly zero energy outside the boundaries. The mathematical description of these laser distributions are presented in Table.1.

TABLE 1. Different laser distribution pattern.

\begin{tabular}{|c|c|}
\hline Top-hat & Dist $=A^{*} \exp \left(-\frac{\left(\max \left(\operatorname{abs}(\xi)-\xi_{0}, 0\right)^{2}+\max \left(\operatorname{abs}(\eta)-\eta_{0}, 0\right)^{2}\right)}{\left(2^{*} \sigma^{2}\right)}\right)$ \\
\hline Gaussian & $\operatorname{Dist}=A^{*} \exp \left(-\frac{\left((\operatorname{abs}(\xi))^{3}+(\operatorname{abs}(\eta))^{3}\right)}{\left(2^{*} \sigma^{2}\right)}\right)$ \\
\hline $\begin{array}{l}\text { Case3 (linear width } \\
\text { skewness (LWS)) }\end{array}$ & $\begin{array}{l}\text { Right }_{\text {margin }}=5^{*} \xi_{0} \quad ; \text { Left }_{\text {margin }}=4^{*} \xi_{0} \quad ; m_{\text {slope }}=\left(\frac{\text { Right }_{\text {margin }} \text {-Left }_{\text {margin }}}{2^{*} \xi_{0}}\right) ; \\
\text { Dist }=A^{*}\left(m_{\text {slope }}\left(x i-x i_{0}\right)+\text { Right }_{\text {margin }}\right) ; \text { for } \xi \in\left[-\xi_{0}, \xi_{0}\right] \text { and } \eta \in\left[-\eta_{0}, \eta_{0}\right]\end{array}$ \\
\hline $\begin{array}{l}\text { Case4 (linear longitudinal } \\
\text { skewness (LLS)) }\end{array}$ & $\begin{aligned} \text { Right }_{\text {margin }} & =5^{*} \eta_{0} \quad ; \text { Left }_{\text {margin }}=4^{*} \eta_{0} \quad ; m_{\text {slope }}=\left(\frac{\text { Right }_{\text {margin }} \text {-Left }_{\text {margin }}}{2 * \eta_{0}}\right) ; \\
\text { Dist } & =A^{*}\left(m_{\text {slope }} *\left(\eta-\eta_{0}\right)+\text { Right }_{\text {margin }}\right) ; \text { for } \xi \in\left[-\xi_{0}, \xi_{0}\right] \text { and } \eta \in\left[-\eta_{0}, \eta_{0}\right]\end{aligned}$ \\
\hline
\end{tabular}

In Table 1, $A$ is an adjusting parameter for each case to produce the same unit energy for all laser distributions. In other words, irradiated area times the intensity is equaled to 1 for all cases. The parameter $\sigma$ is the standard deviation to control how much intensity is gradually decreased. Illustration of these laser distribution patterns with their intensities have been shown in Fig.2. The last two cases will be named as LWS and LLS as abbreviations in rest of the paper. 

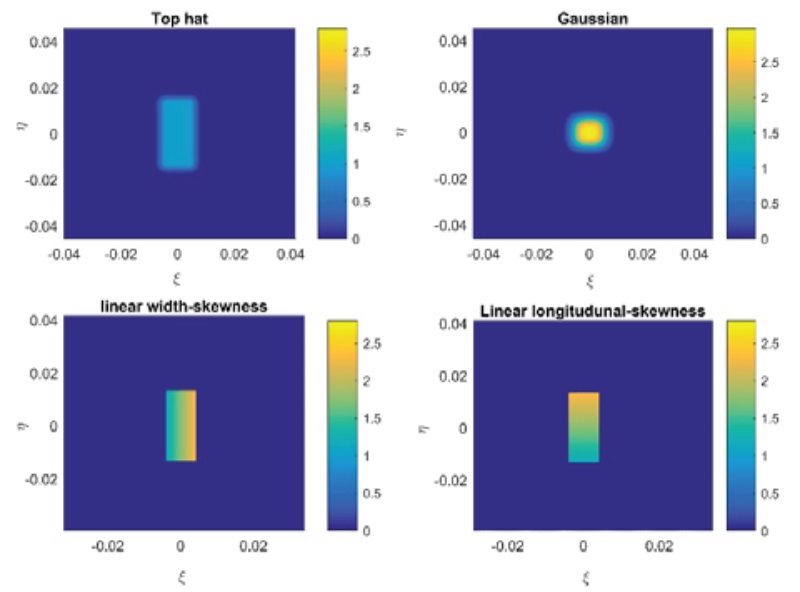

FIGURE 2.Different laser distribution with the same unit input energy.

\section{RESULTS AND DISCUSSION}

The energy distribution charts have been illustrated in Fig.3 to show how input energy is absorbed by the components (tape, laminate, roller) with different AR values and laser patterns. In this figure, 'ref- 0 ' and 'ref-1' indicate without considering reflection, and with considering the first reflection, and 'Total' represents the summation of them. In addition, the 'lost' indicates how much energy is not absorbed by all components. The top-hat laser distribution energy is almost divided equally among components. However, for the Gaussian laser distribution, a large difference in absorbed energy can be observed between the laminate and tape as the laminate receives relatively small amount of energy ( $13-15 \%)$. This is due to the laser position and center of laser light since a larger amount of energy is hit on the tape. Thus, it can be interoperated that laser position is an important factor in the Gaussian distribution. In the Top-hat and Gaussian distributions, since the gradual decrease outside boundaries in laser energy is assumed, the roller receives $\sim 32-36 \%$ of the energy, which is not desired, as it is intended to deliver energy only to the laminate and the tape. For ideal cases which are LLS and LWS, the amount of energy given to the components is approximately similar, however, a little difference $(\sim 0.5 \%)$ in the amount of lost energy is observed. The roller in LLS and LWS receives a smaller amount of input energy $(\sim 16 \%)$ as laser light is concentrated on the center, and no gradual decrease outside the borders is assumed. Changing $A R$ influences the reflection from one surface to another. For $A R=5$, the amount of absorbed energy slightly changes (up to $2 \%$ ), but the interesting point is decreasing the lost energy in all cases $(\sim 3 \%)$. The reason is the geometrical effect in which both laminate and tape have more interaction for passing energy by reflected rays from one surface to another.
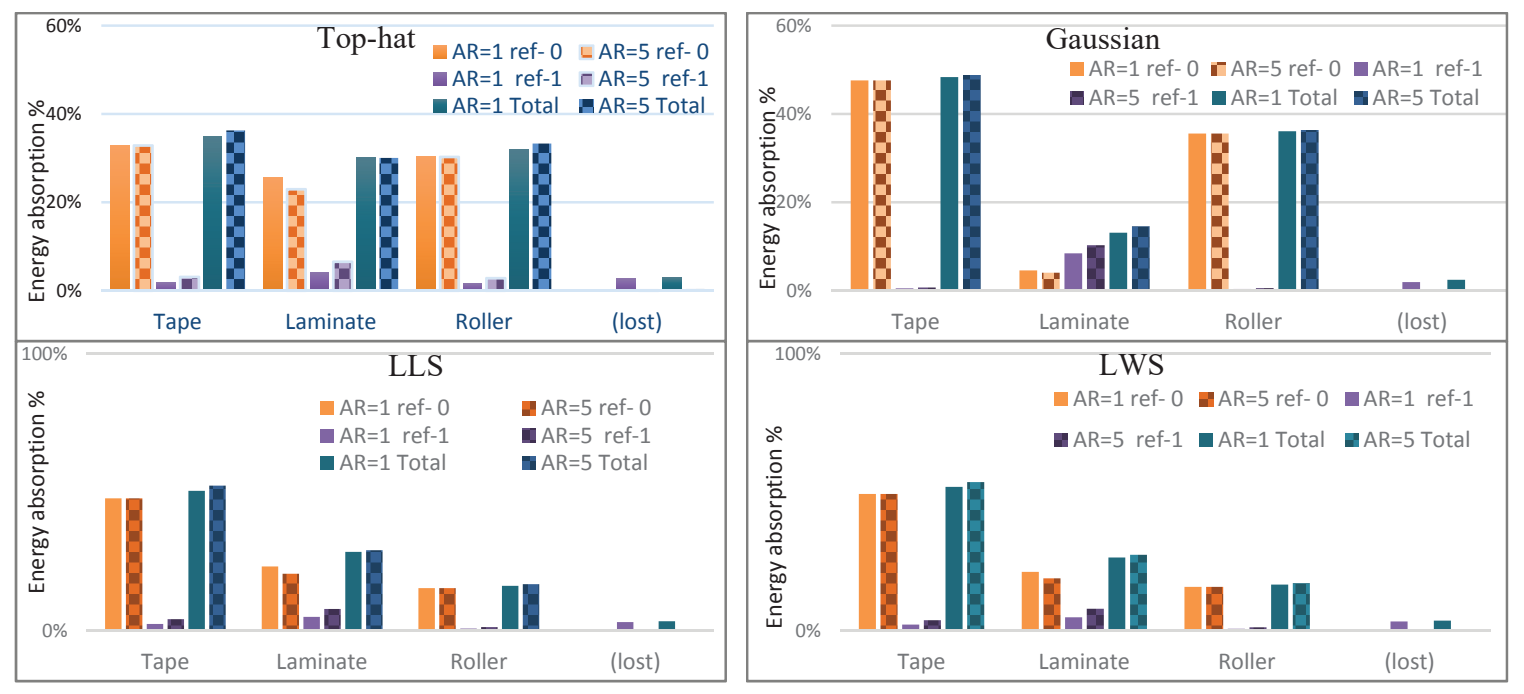

FIGURE 3. Energy absorption of components using the specular reflection of four laser distributions with $A R=1$ and 5 . 
When the non-specular reflection model is employed, some of the reflected rays are scattered in different directions. Fig. 4 shows the absorbed energy after $1^{\text {st }}$ reflection for the specular and non-specular reflection models with $\mathrm{AR}=1$ and 5. As it is seen, the difference between the specular and non-specular models is relatively small. For tape and roller this difference is less than $0.5 \%$, and for the laminate it's less than $2 \%$ in all laser distribution models. Moreover, as it is mentioned before in Fig. 3, the lost energy for all four laser distributions is decreased for $A R=5$ (up to 3\%). This reduction in lost energy for $A R=5$ can be interpreted as geometrical effect of roller and laminate causing more interaction of reflected rays between them. It is worth to mention that the amount of $2^{\text {nd }}$ reflection is less than $0.5 \%$ for all cases, therefore it is not depicted in Fig. 3 and Fig. 4.
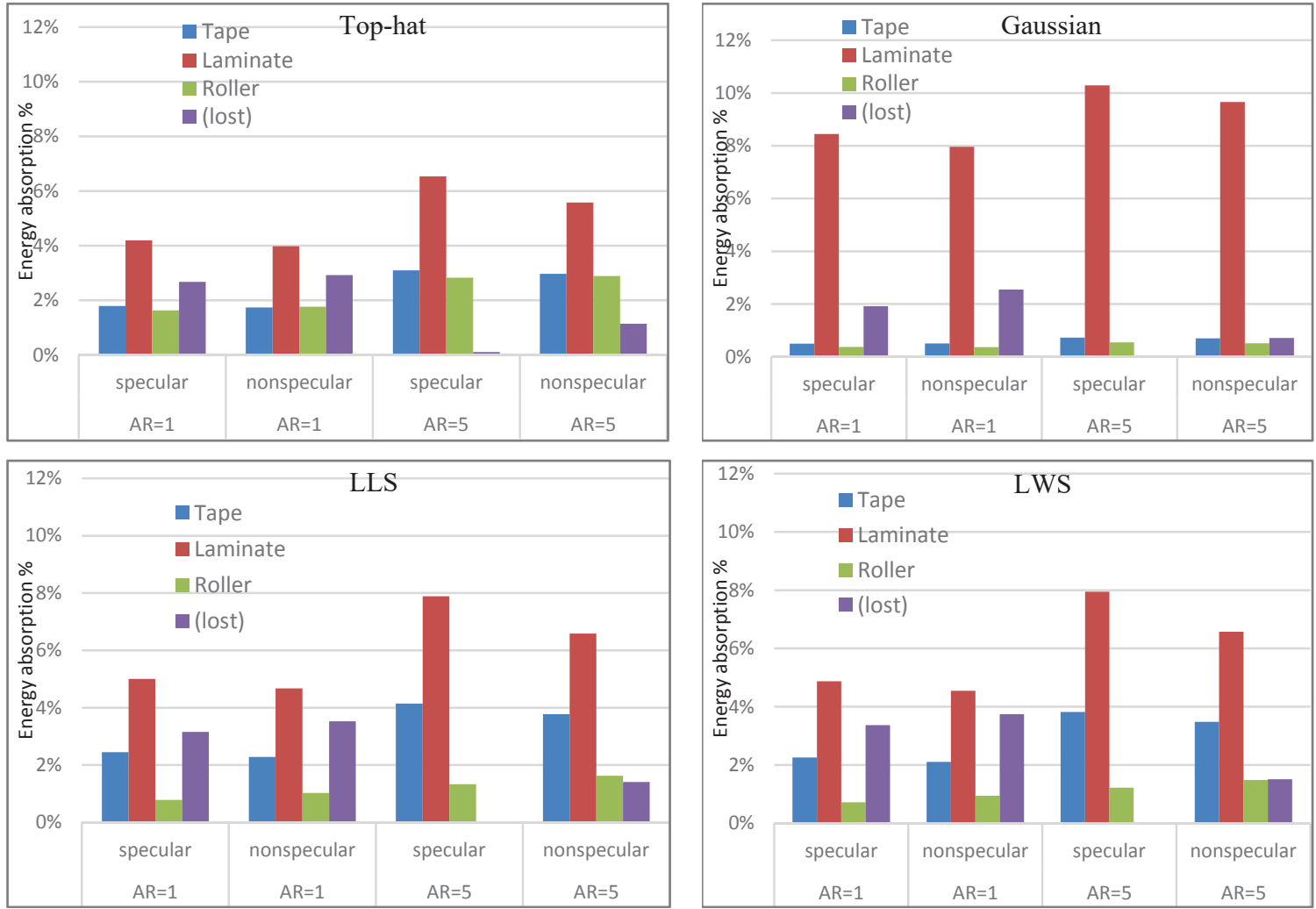

FIGURE 4. Absorbed energy of components from $1^{\text {st }}$ reflection for $\mathrm{AR}=1$ and 5 in specular and non-specular model for four laser distributions. 
The effect of laser distribution on the temperature distribution can be observed on tape and substrate surfaces from Fig.5 and Fig.6. As it is seen in Fig. 5, different temperature distributions are observed with different maximum temperature. For the Top-hat distribution, the energy occupies a larger area, however, in other cases, it is more concentrated in a relatively smaller area. Additionally, the lowest energy is captured for the Gaussian model, because the relatively large amount of energy is emitted into the tape which is seen in Fig. 6. Each tape in Fig. 6 has a different range of temperature distribution. For the Top-hat laser distribution which has the lowest maximum temperature, the temperature difference on the surface is not as much as other cases. On the other hand, Gaussian distribution model has the highest maximum temperature localization among four cases. This illustrates how the location of laser influences the tape temperature. Results of LLS and LWS are comparable and the small difference is observed for the temperature distribution in the middle region of the tapes.
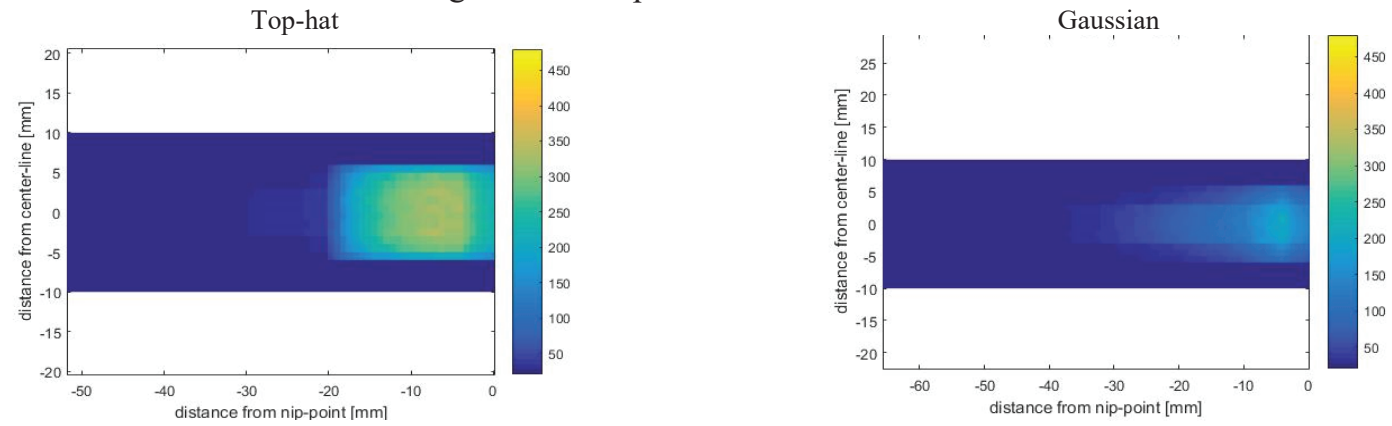

LWS
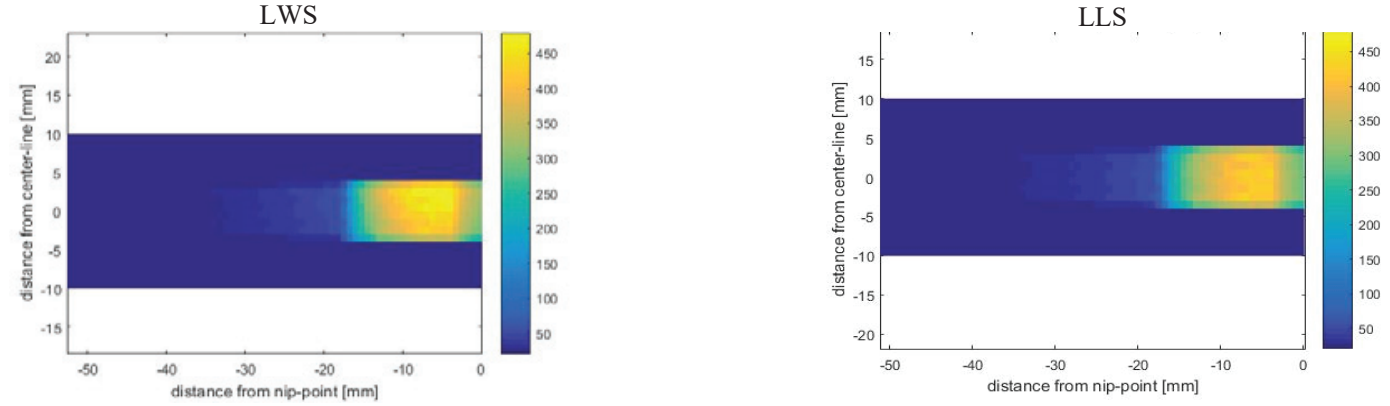

FIGURE 5. Contour plot of temperature distribution $\left({ }^{\circ} \mathrm{C}\right)$ of laminate surface with aspect ratio 1 in specular model.
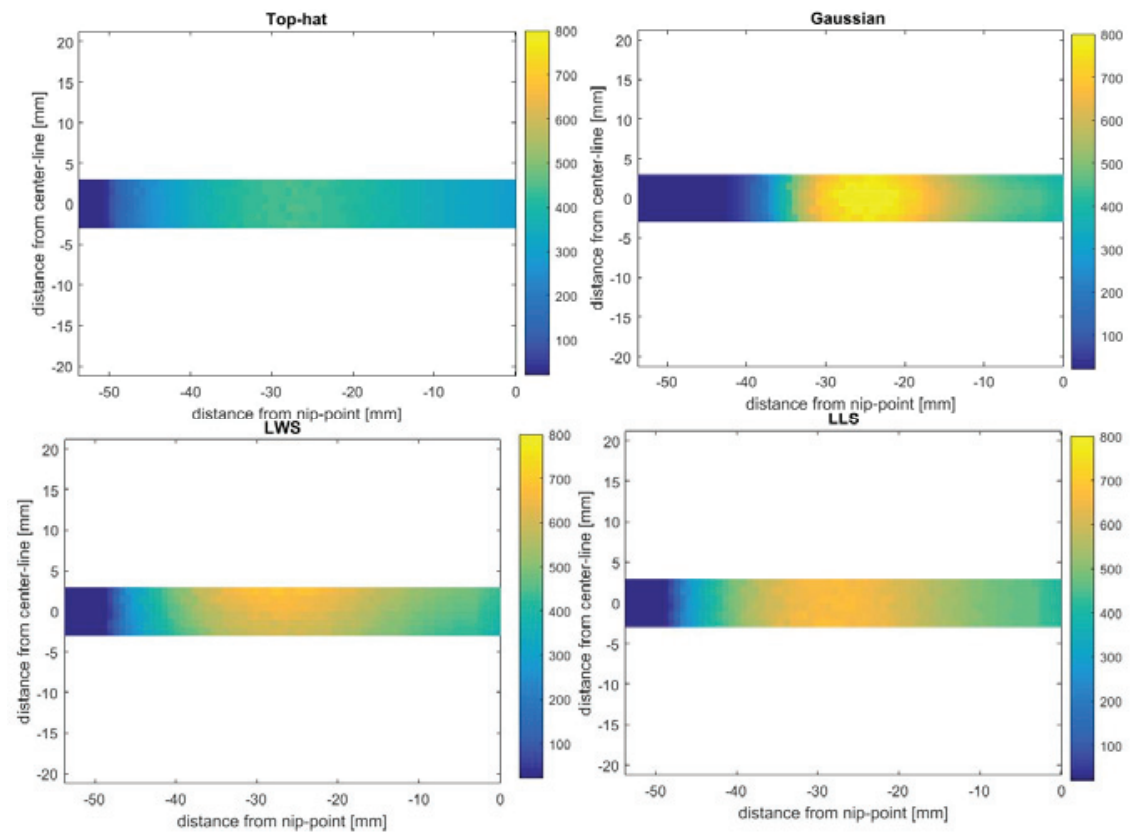

FIGURE 6. Contour plot of temperature distribution $\left({ }^{\circ} \mathrm{C}\right)$ of tape surface for aspect ratio 1 in specular model. 
The nip-point temperatures are shown in Fig.7. As it is seen, increasing the AR from 1 to 5 would increase the nippoint temperature of the tape for all cases with $\sim 5 \%$, however, this change is relatively small ( $1 \%)$ for the Gaussian distribution. For the laminate, this trend is the opposite. It is seen that with an increase in AR, the nip-point temperatures decreases $12-22 \%$, for all distributions excluding the Gaussian distribution. Moreover, the difference between the specular and non-specular results are noticeable $(\sim 8 \%)$. The reason is the width of laminate which is almost four times greater than tape width. Thus, the laminate can receive higher numbers of reflected rays.
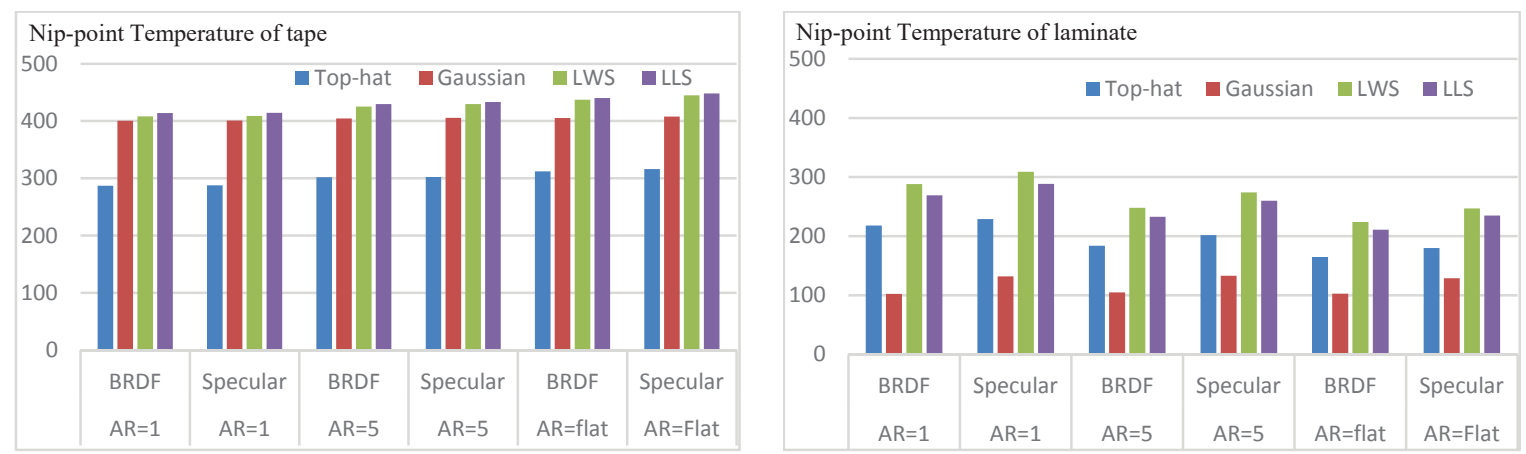

FIGURE 7. Nip-point temperature distribution of tape and laminate in non-specular and specular models for $A R=1,5$, and flat substrate for the laser distributions.

\section{CONCLUSION AND FUTURE WORK}

In the current study, numerical modeling of laser radiated on circular surfaces assuming four laser patterns was investigated. The non-specular reflection model was implemented besides the specular model to consider the effects of the anisotropic reflective behavior of the fiber-reinforced thermoplastic tape. The heat transfer model was combined with the optical model to calculate the tape and laminate temperatures. The nip-point temperature of tape and laminate was predicted considering the effects of geometrical parameters. Furthermore, the quantitative results were represented to show how laser patterns together with geometrical aspect ratios influence energy absorption and nippoint temperature. The geometrical $A R$ influenced nip-point temperature up to $22 \%$ and anisotropy reflection influenced temperature difference up to $8 \%$ for the laminate. However, an optical model considering the non-parallel laser beam toward placement direction seems necessary. It should be able to capture the effect of angle and distance for different laser distributions to obtain better understanding regarding the process parameters for future process optimization.

\section{ACKNOWLEDGMENTS}

The amblifibre project has received funding from the European Union's Horizon 2020 research and innovation program under grant agreement No 678875.

\section{REFERENCES}

1. R. Funck and M. Neitzel, Composites Manufacturing 6, 189 (1995).

2. W.J.B. Grouve, Weld strength of laser-Assisted tape-Placed thermoplastic composites (s.n., S.l., 2012).

3. Y.M. Toso, P. Ermanni, and D. Poulikakos, Journal of Composite Materials 38, 107 (2004).

4. A. Comer, D. Ray, W. Obande, D. Jones, J. Lyons, I. Rosca, R.O. Higgins, and M. Mccarthy, Composites Part A: Applied Science and Manufacturing 69, 10 (2015).

5. A. Yousefpour and M.N.G. Nejhad, Journal of Thermoplastic Composite Materials 14, 129 (2001).

6. C. Stokes-Griffin and P. Compston, Optics and Lasers in Engineering 72, 1 (2015).

7. J.M. Reichardt, I. Baran, and R. Akkerman, ECCM17 - 17th European Conference on Composite Materials, Munich, Germany, 26-30th June 2016. 